\title{
Research on Urban Tourism Communication Strategy of Douyin Short Video
}

\author{
Ying Shi \\ Shanghai Publishing and Printing College, Shanghai, China \\ Email: syfanxiang@163.com
}

How to cite this paper: Shi, Y. (2021) Research on Urban Tourism Communication Strategy of Douyin Short Video. Open Access Library Journal, 8: e7061.

https://doi.org/10.4236/oalib.1107061

Received: November 14, 2021

Accepted: December 17, 2021

Published: December 20, 2021

Copyright $\odot 2021$ by author(s) and Open Access Library Inc.

This work is licensed under the Creative Commons Attribution International License (CC BY 4.0).

http://creativecommons.org/licenses/by/4.0/

\section{(c) (i) Open Access}

\begin{abstract}
In recent two years, the mobile short video Tiktok, represented by the jitter, has attracted wide attention and love from APP. In particular, the novel coronavirus pneumonia epidemic affects the click rate and attention of short videos. Due to certain travel restrictions, short videos with tourism as the theme are particularly popular. Tiktok video is a major strategy for city tourism brand communication. Through case studies, data analysis and longitudinal comparison, this paper affirms the important role of new communication strategies in city tourism and predicts its future development trend.
\end{abstract}

\section{Subject Areas}

Art, Culture, Journalism and Communication, Sociology

\section{Keywords}

New Media, Short Video, City Tourism

\section{Introduction}

With the rapid development of mobile Internet and social media, the spread of short videos has become increasingly popular. As the latest and most popular application in the social field, a short video has gradually become an important platform to spread the image of a city by virtue of its entertainment and impressive expression. Many ordinary citizens use short videos to redefine their city's image. With the participation of different user groups, urban food, life and other market contents are disseminated from different perspectives. Constitute a threedimensional image of the city [1]. Such a special city image has become popular in many tourist cities through the spread of short videos. It has opened up a new road for urban tourism communication. 
Since 2017, Douyin's video of Xi'an bowl smashing has gone viral. Xi'an began to go viral. Xi'an became a web celebrity city from an ancient capital of six dynasties. Subsequently, Hongyadong in Chongqing and Daocheng Yading in Sichuan all started to become popular in Douyin. Douyin has created one web celebrity city after another. Urban tourism has also become an important attribute of Douyin.

\section{Communication Advantages of Short Video City Tourism}

According to the information on Ctrip in 2019, Xi'an, Xiamen, Henan and other places were called short daily attendance. Especially in the first half of 2020, our country encountered COVID-19, and the tourism market of the entity did not gradually open until October. According to the new tourism data, the click rate of short videos related to tourism remained high, and people paid attention to 3 times the number of people in 2019. Relying on the dissemination of short videos, tourism delicacies and tourist souvenirs in the above areas were sold online, and the sales volume increased by 10 percentage points. It has to be said that it is a new miracle of Communication advantages of short video city tourism.

\subsection{Douyin Gives More Cities a Chance to Show Their Image to the Public. Chongqing, Xi'an and Xiamen were among the Top Three Cities in the Video Streaming Ranking of Douyin City}

These three cities rely on their own web celebrity scenic spots based on the rapid spread of douyin, high-quality content can play the dissemination value, while douyin platform hot style caused by imitation and follow the trend, the birth of a batch of new urban web celebrity elements, to achieve bottom-up reconstruction or upgrade of city image. On the one hand, users make use of douyin special effects and background music to display tourist attractions in a new way, and endue the city with new vitality in a form favored by young people. For example, the songs of Xi'an people and numerous historic buildings in Xi'an break the public's old stereotype of Xi'an [2]. On the other hand, the new urban elements created by Douyin replace the traditional scenic spots to become a new tourist punching place. The web celebrity hotel in moganshan, which has become popular in douyin, and the liziba station light rail in chongqing, which passes through the building, have become new gathering places for young people to clock in. Douyin is a new fashion for young people to record their travels and discover the beauty in their lives. This emerging behavior has also brought new ideas of urban tourism communication to many cities with low popularity and relatively deficient tourist attractions.

\subsection{Douyin Vividly Demonstrates the Connotation of City Image with Short Videos More in Line with Young People's Expression Methods and Aesthetic Habits, and Becomes an Important Platform for the Dissemination of City Image}

Compared with pictures and words, video has more advantages in information 
transmission. Different from films and other artistic and professional video works, short videos represented by Douyin have a low production threshold and are an expression of ordinary People's Daily life through videos. In the era of short videos on mobile terminals, ordinary citizens have become the definers of urban image. In the traditional media era, the government is the definer and communicator of the city image. In the era of mobile Internet, citizens have the right to speak and enrich the specific connotation of city image in the subdivision fields such as urban tourism, culture and food. Compared with the official accounts of the government, ordinary citizens spontaneously tell city stories through short videos and become the backbone of shaping city business cards [3]. Douyin users are both receivers and disseminators of information. With the popularization of intelligent devices and the convenient operation of Douyin, the occurrence and propagation of events can be carried out almost simultaneously.

\subsection{Through the Synchronous Transmission of Sound and Picture, Short Videos Will Bring Strong Impact to the Audience's Visual and Auditory Sense}

Background music with strong sense of rhythm enhances the appeal of content transmission. Therefore, ordinary citizens are more willing to participate in the creation of content creation, and can record the details of urban life anytime and anywhere. At the same time, citizens in the process of participating in the creation of content will also enhance the sense of identity to the city.

\section{Communication Strategy of Short Video City Tourism}

\subsection{Flexible Use of Douyin to Spread Urban Tourism}

The city of Xi'an became the first batch of web celebrity cities on douyin due to its bowl smashing wine on duan yongxingfang. Subsequently, Xi'an seized the opportunity to dominate the number of douyin city's travel videos through the later period of brush pastry, Xi'an people's songs and Datang Buyecheng. In the same period, the song "Nanjing, Nanjing" was performed by Wang Leehom in Nanjing concert [4]. Also in the tremolo the fire. The beautiful melodies of Nanjing, accompanied by videos of popular scenic spots such as Nanjing Zijin Mountain, Xinjiekou and Confucius Temple, have been streamed by Douyin skyrocketing, attracting many tourists to visit Nanjing. But Nanjing did not seize the moment to launch a new hot spot. After the early end of the urban hot spot, the heat of Nanjing city gradually declined. Nanjing is now no longer a web celebrity city. Therefore, it is necessary to master the rhythm in urban tourism communication. When there are hot spots in urban tourism, the government should not only give reasonable guidance, but also consider the sustainable development after the hot spots fade away.

\subsection{Reasonably Control the Time of Short Videos}

Grasp the reasonable time of short video, sometimes it will get twice the result 
with half the effort. According to the latest iResearch Market Research Report in 2020, after data calculation, short videos between 30 seconds and 90 seconds are more popular, and the first 8 seconds of short videos can capture the prime time of the audience.

Part of the reason short videos are so popular today is that the pace of life is accelerating and people are demanding faster and more concise access to information. Therefore, the ability of short videos to catch people's attention in the first few seconds becomes particularly critical. Therefore, in the process of short video shooting and editing, it is necessary to avoid the phenomenon of making a false impression, and to hit the key point and show the most attractive places in the city tourism first. This will give the user to continue to look at the interest. Chongqing, for example, is using short videos to promote city tourism. A subway that flies through the floors. Hongya Cave lit up at night. This kind of scenery is the easiest to give the intuitive feeling. [5]

\subsection{Grasp the Video Playback Speed}

Video playback speed is also one of your city's business cards. For example, when you click on the short promotional video of Suzhou city, you will find the short video of Suzhou is short but slow. It slowly shows you the landscape of a garden or the boats slowly moving on the ancient town. But when you get to the Shanghai city promotion short video. What you see more is the light show of the Lujiazui complex in front of your eyes quickly from morning till night. Because Suzhou is different from Shanghai in positioning, Suzhou as a water town in the south of the Yangtze River, everything is slow and steady [6]. As an international financial center, Shanghai's fast pace of life shows the vibrant urban pace of life. Because you need to be clear about the hotspot location that your city wants to express to determine the speed of video playback

\section{Suggestions of Douyin Short Video on Urban Tourism Communication}

\subsection{Attach Importance to the Review of Douyin Short Video Content}

As for short videos themselves, they should play the role of gatekeeper in terms of content, eliminate false, violent and other bad content, and actively optimize the system, strictly check the content, and improve the content inspection process. Strengthen the management of the platform. Create a video platform with positive energy.

\subsection{Strengthening Guidance of Public Opinion}

When building the image of city tourism, the public opinion must be supervised. Make short videos the new frontier in the battle for public opinion [7]. Let the general public and people from all walks of life see the government's sincerity in controlling public opinion, can show the public and the outside world a strong government credibility. 


\section{Conclusions}

At present, the communication advantages of a short video are still unabated. Douyin and other short video APP are gradually called people's daily companions and trusted partners. Short videos are also called for a power that can not be ignored for the shaping and dissemination of city images.

In the future, no matter developing online tourism resources, users can enjoy the great scenery of the motherland without leaving home, and give users an immersive experience through digital twins; or in offline tourism, it undertakes the functions of guidance and service. The short video will be called the continuous driving force for the sustainable development of urban tourism.

\section{Conflicts of Interest}

The author declares no conflicts of interest.

\section{References}

[1] Chen, Y.Y. and Lv, Y.X. (2018) Tiktok's Presentation and Dissemination of City Tourist Attractions. Audio Visual Circles. Radio and Television Technology, p. 117-119.

[2] Qian, J.X. (2018) The Tiktok Brings “Travel”. Tourism Overview, p. 17-19.

[3] Chen, Y.H. (2018) Marketing Awakening of Online Red Cities. Western Development, p. 16-19.

[4] Xie, H. (2018) Discussion on the Successful Model of We Media Video Advertising Marketing-Taking “One Piece” Video as an Example. Modern Marketing, p. 59.

[5] Gao, R. (2018) Analysis of the Marketing Role of Mobile Short Video. TV Guide, p. 253-254.

[6] Dai, X.Y. (2018) Analysis of the Tiktok Short Video APP Tourism Marketing Reference Significance. Communication Force Research, p. 117.

[7] Wang, J.S. (2018) Research on the Development Status and Trend of Online Video Marketing. Productivity Research, p. 118-122. 